\title{
HLA-Cw6 Allele Expression is Associated with Good Narrowband Ultraviolet B Response in Javanese-Indonesian Psoriasis Subjects
}

\author{
Thianti Sylviningrum ${ }^{1, *}$, Ismiralda Oke Putranti ${ }^{1}$, Octavia Permata Sari ${ }^{2}$, \\ Fitranto Arjadi $^{3}$, Triasari Oktavriana ${ }^{4}$ \\ ${ }^{1}$ Department of Dermatology and Venereology, Faculty of Medicine, Universitas Jenderal Soedirman, J1. Dr. Gumbreg No.1, \\ Purwokerto 53112, Indonesia \\ ${ }^{2}$ Department of Parasitology, Faculty of Medicine, Universitas Jenderal Soedirman, Jl. Dr. Gumbreg No.1, Purwokerto 53112, Indonesia \\ ${ }^{3}$ Department of Anatomy, Faculty of Medicine, Universitas Jenderal Soedirman, Jl. Dr. Gumbreg No.1, Purwokerto 53112, Indonesia \\ ${ }^{4}$ Department of Dermatology and Venereology, Faculty of Medicine, Universitas Sebelas Maret, Jalan Ir. Sutami 36A, Surakarta 57126, Indonesia \\ *Corresponding author. E-mail: thianti.sylviningrum@unsoed.ac.id
}

Received date: Jan 12, 2021; Revised date: Aug 17, 2021; Accepted date: Aug 20, 2021

\section{Abstract}

$\mathrm{B}$ ACKGROUND: Psoriasis is an autoimmune disease involving genetic-environmental factors. Human Leukocyte Antigen (HLA)-Cw6 allele is the main genetic risk factor of psoriasis, but its prevalence varies widely. HLA-Cw6 allele correlates with psoriasis clinical type and treatment responses. Narrowband ultraviolet B (NB-UVB) and methotrexate (MTX) are effective psoriasis treatments but their association with HLA-Cw6 allele has not been identified. The study aims to determine the association between HLA-Cw6 allele expression, NB-UVB, and MTX treatment responses in Javanese-Indonesian psoriasis subjects.

METHODS: Ninety Javanese-Indonesian psoriasis subjects were recruited in this study, 45 subjects were treated using NB UVB, while the other 45 subjects were treated using MTX, respectively, for 12 weeks. The psoriasis diagnosis and treatment responses were evaluated by dermatologists using Psoriasis Area Severity Index (PASI) score. The HLA-Cw6 allele was examined using the single-specific-primer polymerase chain reaction method. Fisher's Exact and Chi-square tests were employed, where $p$-value $<0.05$ was considered of significant association.

RESULTS: The HLA-Cw6 allele positivity was identified in 23 psoriasis subjects $(25.56 \%)$, while the other 67 subjects expressed HLA-Cw6 allele negative (74.44\%). Female with HLA-Cw6 allele positivity who did not have comorbid disease show good response to NB-UVB than MTX. Meanwhile, subjects who were treated with MTX showed no association between therapeutic response and HLA-Cw6. HLA-Cw6 status was not correlated with the onset of psoriasis, family history, and comorbid diseases in all subjects.

CONCLUSION: HLA-Cw6 allele expression is more associated with good NB-UVB treatment response than with MTX treatment response in female Javanese-Indonesian psoriasis subjects without comorbid disease.

KEYWORDS: HLA-Cw6, narrowband ultraviolet B, methotrexate, Javanese, psoriasis

Indones Biomed J. 2021; 13(3): 324-31

\section{Introduction}

Psoriasis is an autoimmune disease marked with erythematous scaly plaques with demarcated borders from normal skin.(1) The World Health Organization mentions that psoriasis patients may be up to $0.09-11.4 \%$ worldwide. (2) Meanwhile, psoriasis prevalence in Indonesia is difficult to determined, but the tendency showed enhancement as found from 400 (2017) to 458 (2018) psoriasis patients visits in Sanglah Hospital, Bali.(3) Psoriasis patients may show declining quality of life and have a long term 
treatment, that may be associated with low compliance and high-cost therapy.(2) By clinical manifestation, the plaque type psoriasis can be found in daily practices the most. The plaque type psoriasis patients may show decreasing quality of life and need a long term treatment, that is associated with lower compliance and higher side effects.(2)

In psoriasis, there are complex interactions between genetic and environmental factors. The complexity of genetic factors involved in psoriasis may contribute to different clinical types and treatment responses.(4) Therefore, identification factors, including pharmacogenetic biomarkers, are necessary to predict therapeutic response in psoriasis patients. Environmental factors such as infection; trauma; obesity; lifestyle such as alcoholism, smoking; psychological complaints like depression, anxiety, suicidal attempts, and stress, meanwhile, may affect the severity of psoriasis.(5,6) Major histocompatibility complex class I molecule, namely HLA-Cw6 allele, is the strongest genetic factor associated with psoriasis.(1) The allele is expressed on the surface of keratinocytes and dendritic cells. The HLA-Cw6 allele makes it easier for keratinocytes and dendritic cells to present antigens to naive $\mathrm{CD} 8^{+} \mathrm{T}$ cells. Activated naive $\mathrm{CD}^{+} \mathrm{T}$ cells can be differentiated into $\mathrm{Tc} 17 \mathrm{CD} 8^{+}$cells that are capable of releasing Interleukin (IL)-17.(7) The IL-17 may increase neutrophils recruitment from vascular to epidermis and trigger inflammation that can worsen psoriasis.(8) The IL-17 together with other cytokines from different pathways, namely tumor necrosis factor (TNF)- $\alpha$, interferon (IFN)- $\alpha$, IFN- $\gamma$, IL-1, and IL6 , may affect atherogenesis. This condition may worsen because IL-17/neutrophils axis contributes to atherogenesis and leads to cardiovascular diseases.(6) Comorbid diseases commonly found in psoriasis include cardiovascular disorders, obesity, diabetes, metabolic syndrome, psoriatic arthritis, gastrointestinal disorders, etc. These comorbid diseases may increase the mortality of psoriasis patients.(6)

HLA-Cw6 can be found in a psoriasis patient or a healthy person, but its distribution among race and ethnicity may differ. HLA-Cw6 allele expression has a high prevalence in Caucasians from $35.2 \%-36.7 \%$ in Brazil to $56.0 \%-77.2 \%$ in Poland. Lower prevalence of HLA-Cw6 allele is found from $10.5 \%-12.0 \%$ in Japan to $67.6 \%-71.1 \%$ in India.(5) According to Indonesian Central Bureau of Statistics in 2010, Javanese people is the biggest ethnic populations with 95.2 million people (40.2\%) who mostly live in Java Island.(9) Previous study shows that $22 \%$ out of 60 Javanese-Indonesian psoriasis patients express HLACw6 allele without any association with early-onset, clinical type of psoriasis, and disease severity.(10) However,
HLA-Cw6 allele is also associated with type 1 psoriasis characterized with early-onset $(<40$ years old); familial history; and more severe disease.(5) This allele may also affect psoriasis disease course, phenotypic manifestations, severity of the disease, comorbid diseases and therapeutic response.(11)

Phototherapy using NB-UVB 311-313 nm and MTX are highly available and feasible psoriasis treatment methods in Indonesia compared to that with biological agents. HLA-Cw6 allele is associated with good responses and minimal side effects in psoriasis subjects treated with MTX (12), but its association with NB-UVB phototherapy remains unknown. MTX may cause $\mathrm{T}$ cells activated apoptosis through dihydrofolate reductase inhibition so that DNA synthesis is inhibited.(13) There are several acute side effects associated with MTX treatment such as abdominal pain, nausea, diarrhea, loss of appetite, and liver fibrosis as chronic adverse events.(13) DNA damage, cellular cell injury and reactive oxygen species creation are the causes of $\mathrm{T}$ cells and keratinocytes apoptosis by NBUVB phototherapy.(14) NB-UVB shows lower systemic effect than MTX since the ultraviolet only reaches skin layers. To the best of our knowledge, this is the very first study to assess the association of HLA-Cw6 status with NBUVB which compares it with MTX therapeutic response in Javanese-Indonesian psoriasis subjects. This study aims at identifying the association of HLA-Cw6 allele expression with NB-UVB and MTX treatment responses in JavaneseIndonesian psoriasis patients. Hopefully, the result of this study will enhance the importance of pharmacogenetic in psoriasis management in the future.

\section{Methods}

\section{Sample Collection}

From the whole psoriasis subjects, we had included Javanese-Indonesian psoriasis subjects affected by moderate to severe (PASI score $\geq 10$ ) plaque psoriasis with Fitzpatrick's skin type III-IV (15) who had been treated with NB-UVB or MTX for 12 week of observation period. The PASI score was used to measure the severity of psoriasis on the erythema, scaling and thickness of the skin lesions. Subjects who failed the follow-up during the 12-week observation and disagreed to participate in this study were excluded. The subjects were not allowed to have any other systemic medication, while only emollients, $3 \%$ salicylic acid maximally in concentration, $0.25 \%$ desoxymethasone cream/topical steroid ointment, and a combination of both 
agents were allowed for their treatment. Therefore, our study involved totally 90 subjects selected from 2 hospitals in Central Java, who were equally divided into 2 groups. Group A consisted of 45 subjects enrolled in Universitas Sebelas Maret Hospital, Surakarta, who were only treated with NB-UVB once a week based on the modification of another study.(16) The other 45 subjects were obtained from Margono Soekarjo Public Hospital, Purwokerto who were treated with MTX according to the procedures explained in the previous study.(17)

This study was conducted from March 2019 to February 2020. The Health Research Committee, Faculty of Medicine, Universitas Jenderal Soedirman had approved this study under Approval Number: Kept.161/UN23/14/ PN.01.00/2019.

\section{Procedures of MTX and NB-UVB Treatments}

A $311 \mathrm{~nm}$ NB-UVB phototherapy cabinet (Daavlin Company, Ohio, USA) was employed in this study with a starting dose of $500 \mathrm{~mJ} / \mathrm{cm}^{2}$ once a week as modification in initial dose and the frequency of therapy from previous study.(16) According to another study, an increase of $20 \%$ from the previous dose up to $2977 \mathrm{~mJ} / \mathrm{cm}^{2}$ as the modified maximum dose weekly, and dose adjustment was made when the clinicians found erythematous skin as the phototherapy side effect.(18) We used a $2.5 \mathrm{mg} /$ tablet MTX (Rheu-Trex, Kalbe, Jakarta, Indonesia) in this study. Based of previous study, MTX was administered to the subjects with initial dose 7.5-10 mg per week, and increased by 2.5 $\mathrm{mg}$ weekly up to $15 \mathrm{mg} /$ week as the maximum dose.(17) In case of MTX adverse effects evaluation, abnormal routine hematologic and blood chemistry tests, or elevated liver enzymes over 3-fold normal value, the MTX treatment was stopped.(17) Supplemented folic acid was also administered to the subjects daily except they day they consume MTX. All treatment responses from NB-UVB phototherapy and MTX were evaluated in weeks 1 and 12 by dermatologists using PASI score criteria from another study.(16)

A good therapeutic response was established when there was improvement in psoriasis severity based on a $75 \%$ decrease of PASI score at week 12 compared to week 1. Poor therapeutic response was determined in case of worsened disease severity as marked with increasing PASI score or the PASI score remained the same at week 12 compared to week 1. Both therapeutic responses were evaluated by 2 certified dermatologists. Subjects' demographic characteristics such as the onset of psoriasis; gender; familial history from first- and second-degree relatives; baseline PASI score, existing comorbid diseases and patient's complaints during treatment were obtained through questionnaires and medical records.

\section{DNA Isolation and HLA-Cw6 Allelic Analysis}

HLA-Cw6 allele was identified through DNA isolation steps, quantitative DNA test, and HLA-Cw6 allelic analysis. (10) DNA was isolated from subjects' $3 \mathrm{~mL}$ peripheral venous blood following the procedures in the PureLink Genomic DNA Kit instructions (Invitrogen, Waltham, MA, USA). Quantitative DNA test was conducted using Qubit fluorescence spectrophotometry (Thermo Fisher Scientific, Waltham, MA, USA) in the Research Laboratory, Faculty of Medicine, Universitas Jenderal Soedirman, Indonesia, to determine the concentration and purity of the isolated DNA. The HLA-Cw6 allelic analysis employed the single-specificprimer polymerase chain reaction (SSP-PCR) method. Allele was amplified using the allele-specific (297 base pairs) forward primer:TACTACAACCAGAGCGAGGAGGTCG; reverse primer: CAGCCATACATCCA, while the positive control on 618 base pairs forward primer: TTGAGGATTCTCCACTCCCCTGAGCTG; reverse primer: TGCCTGGCGCTTGTACTT employed Applied Biosystem PCR (Thermo Fisher Scientific, Waltham, MA, USA).(19) The 297 base pairs showed HLA-Cw6 allele specificity without differentiating homozygote and heterozygote status. The PCR was conducted for $2 \mathrm{~min}$ at $96^{\circ} \mathrm{C}$, followed with 30 cycles of amplification for $25 \mathrm{sec}$ at $96^{\circ} \mathrm{C}, 1 \mathrm{~min}$ at $60^{\circ} \mathrm{C}$, and $2 \mathrm{~min}$ at $72^{\circ} \mathrm{C}$. The final extension step was performed for $5 \mathrm{~min}$ at $72^{\circ} \mathrm{C} .(10)$

\section{Statistical Analysis}

The data were analyzed using SPSS (IBM Coorporation, Armonk, NY, USA) with $p$-value $<0.05$ considered as a significant association between variables and confidence interval was given. Fisher's Exact Test and Chi square test were employed in this study to evaluate the association of HLA-Cw6 expression with the characteristics of psoriasis subjects and therapeutic responses.

\section{Results}

All of the 90 Javanese-Indonesian psoriasis subjects participated in this study. The subjects were comprised of 45 psoriasis subjects treated with NB UVB in group A and 45 psoriasis subjects treated with MTX in group B. Subjects with positive HLA-Cw6 allele were all marked in 297 base pairs band, while positive control was identified in 618 base pairs (Figure 1). 


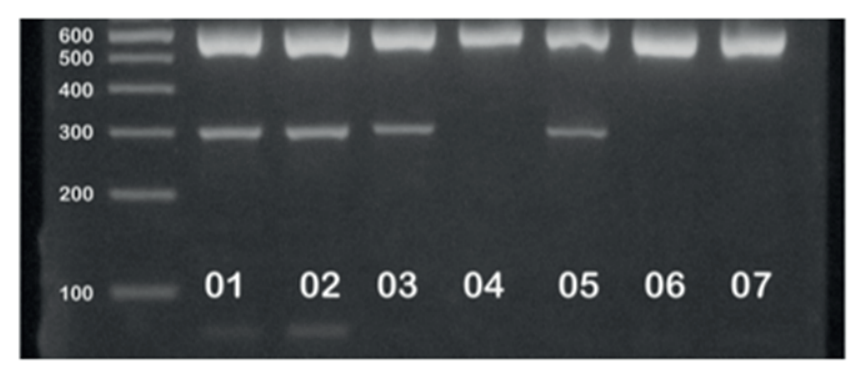

NB-UVB Group (A)

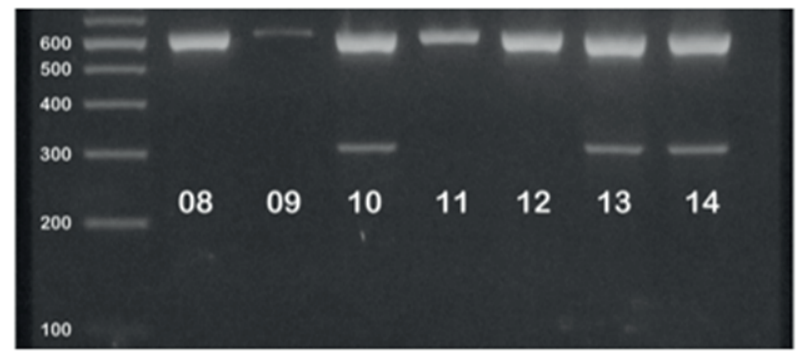

MTX Group (B)

Figure 1. Positive HLA-Cw6 allele is expressed in 297 base pairs band. A: Subjects no. 1, 2, 3, 5 showed positivity HLA-Cw6 allele as representatives from NB-UVB group. B: Meanwhile, subjects no. 10, 13, 14 from MTX group represented positive for HLA-Cw6 allele. Positive control as a SSP-PCR reaction is identified at 618 base pairs.

Based on Table 1, in all psoriasis subjects there was no association of gender, familial history and comorbid diseases with HLA-Cw6 allele positivity ( $p>0.05)$. HLA-Cw6 allele was also expressed by $12(26.66 \%)$ psoriasis subjects in group A and 11 (24.44\%) in group B (Table 2, Table 3). Based on medical records, 11 subjects with positive HLACw6 showed good NB-UVB therapeutic response with mean PASI score of 3 at week 12 from the baseline PASI score of 12. Only one patient with positive HLA-Cw6 with anemia as the comorbid disease showed poor NB-UVB therapeutic response. Contradictorily, NB-UVB phototherapy presents negative HLA-Cw6, with 13 as the mean the baseline PASI score, decreasing to 6 , which was lower than $75 \%$ of PASI score. Comorbid diseases, meanwhile, were found more in subjects with negative HLA-Cw6 than in subjects with positive HLA-Cw6. A significant association was found of positive HLA-Cw6 allele with female gender ( $p=0.006$, $\mathrm{CI}=1.16-2.32)$ and good response in psoriasis subjects under NB UVB therapy $(p<0.033, \mathrm{CI}=0.485-0.904)$ as shown in Table 2. Meanwhile, there was no significant difference between HLA-Cw6 positivity and the onset of psoriasis, familial history, and comorbid diseases. Regardless of HLA-Cw6 positivity, 18 subjects (40.00\%) under NB UVB were reported to have side effects such as pruritus, xerotic skin, and hyperpigmented skin. Interestingly, 3 psoriasis subjects showing positive HLA-Cw6 intending to have MTX treatment previously had nausea and abdominal pain during MTX in the initial dose exposure. The result of our study shows that female Javanese-Indonesian psoriasis subjects with positive HLA-Cw6 treated with NB UVB showed a good response. In group B, there were 6 psoriasis subjects with HLA-Cw6 positivity with a reduction of

Table 1. Association of HLA-Cw6 allele with Javanese-Indonesian psoriasis subjects.

\begin{tabular}{|c|c|c|c|c|}
\hline \multirow[t]{2}{*}{ General Characteristics } & \multicolumn{2}{|c|}{$\begin{array}{c}\text { HLA-Cw6 } \\
\text { n (\%) }\end{array}$} & \multirow[t]{2}{*}{$p$-value } & \multirow{2}{*}{$\begin{array}{c}\text { Confidence } \\
\text { Interval }\end{array}$} \\
\hline & Positive & Negative & & \\
\hline Psoriasis subjects & $23(25.56)$ & $67(74.44)$ & & \\
\hline \multicolumn{5}{|l|}{ Gender } \\
\hline Male & $9(20.45)$ & $35(79.55)$ & $0.278^{\dagger}$ & $0.324-1.392$ \\
\hline Female & $14(30.43)$ & $32(69.57)$ & & \\
\hline \multicolumn{5}{|l|}{ Family history } \\
\hline Positive & $6(42.86)$ & $8(57.14)$ & $0.178^{*}$ & $0.918-3.999$ \\
\hline Negative & $17(22.37)$ & $59(77.63)$ & & \\
\hline Mean age & 44.48 & 46.69 & & \\
\hline \multicolumn{5}{|l|}{ Comorbid diseases } \\
\hline Positive & $4(4.44)$ & $17(18.89)$ & $0.742^{\dagger}$ & $0.532-2.430$ \\
\hline Negative & $19(21.11)$ & $50(55.56)$ & & \\
\hline \multicolumn{5}{|l|}{ Treatment for psoriasis subjects } \\
\hline Methotrexate & $11(24.44)$ & $34(75.56)$ & & \\
\hline NB UVB & $12(26.67)$ & $33(73.33)$ & & \\
\hline
\end{tabular}

*Fisher's exact test, ${ }^{\dagger}$ Chi square test. 
Table 2. Characteristics of Javanese-Indonesian psoriasis subjects treated with NB-UVB (group A).

\begin{tabular}{|c|c|c|c|c|}
\hline \multirow[t]{2}{*}{ Subject Characteristics } & \multicolumn{2}{|c|}{$\begin{array}{c}\text { HLA-Cw6 } \\
\text { n (\%) }\end{array}$} & \multirow[t]{2}{*}{$p$-value } & \multirow{2}{*}{$\begin{array}{c}\text { Confidence } \\
\text { Interval }\end{array}$} \\
\hline & Positive (n=12) & Negative $(n=33)$ & & \\
\hline \multicolumn{5}{|l|}{ Age at psoriasis onset (years old) } \\
\hline$<40$ & $8(17.78)$ & $17(37.78)$ & $0.366^{\dagger}$ & $0.562-4.556$ \\
\hline$\geq 40$ & $4(8.89)$ & $16(35.56)$ & & \\
\hline \multicolumn{5}{|l|}{ Gender } \\
\hline Male & $1(2.22)$ & $18(40.00)$ & $0.006^{\dagger}$ & $1.162-2.321$ \\
\hline Female & $11(24.44)$ & $15(33.33)$ & & \\
\hline \multicolumn{5}{|l|}{ Family history } \\
\hline Positive & $4(8.89)$ & $5(11,11)$ & $0.219^{*}$ & $0.772-5.184$ \\
\hline Negative & $8(17.78)$ & $28(62.22)$ & & \\
\hline \multicolumn{5}{|l|}{ Comorbid diseases } \\
\hline Positive & $1(2.22)$ & $9(20.00)$ & $0.246^{*}$ & $0.047-2.176$ \\
\hline Negative & $11(24.44)$ & $24(53.33)$ & & \\
\hline \multicolumn{5}{|l|}{ Types of comorbid diseases } \\
\hline Anemia & $1(10.00)$ & $1(10.00)$ & & \\
\hline Hypertension & $0(0)$ & $2(20.00)$ & & \\
\hline Diabetes mellitus & $0(0)$ & $4(40.00)$ & & \\
\hline Cellulitis & $0(0)$ & $1(10.00)$ & & \\
\hline HIV infection & $0(0)$ & $1(10.00)$ & & \\
\hline \multicolumn{5}{|l|}{ Adverse events } \\
\hline Positive & $6(13.33)$ & $12(26.67)$ & $0.499 *$ & $0.573-3.924$ \\
\hline Negative & $6(13.33)$ & $21(46.67)$ & & \\
\hline \multicolumn{5}{|l|}{ NB UVB therapeutic response } \\
\hline Good & $11(37.93)$ & $18(62.07)$ & $0.033^{*}$ & $0.485-0.904$ \\
\hline Poor & $1(6.25)$ & $15(9.75)$ & & \\
\hline
\end{tabular}

*Fisher's exact test, ${ }^{\dagger}$ Chi square test.

PASI score from 13 at the baseline to 3 at week 12 . This PASI score reduction, within $75 \%$, is interpreted as a good MTX therapeutic response. The same interpretation of good MTX therapeutic response was observed in negative HLA-Cw6 subjects with decreasing PASI score of 14 at the baseline to 3 PASI score at week 12 . There was no significant difference between HLA-Cw6 positivity and MTX therapeutic response ( $p=1.000, \mathrm{CI}=0.342-2.692)$. Comorbid diseases were more commonly found in MTX treated psoriasis subjects than in subjects under NB UVB therapy. No significant difference was observed between HLA-Cw6 status and adverse events during MTX treatment. Adverse event of mild level nausea was exempted from MTX treated psoriasis subjects, but the effect ceased since MTX was reduced. The study result concludes that positive HLA-Cw6 allele expression is not found associated $(p>0.05)$ with onset, gender, familial history, comorbid diseases, MTX adverse events and therapeutic response (Table 3).

\section{Discussion}

Pharmacogenetics in psoriasis develops recently because of increasing attempts to manage psoriasis subjects in a comprehensive manner. HLA-Cw6 allele is known as the strongest genetic factor in association with psoriatic clinical manifestation and therapeutic responses in Caucasians.(5) However, the contribution of this allele to Javanese-Indonesian psoriasis therapeutic response is not yet elaborated. In Indonesia, in particular, with Javanese people as the dominant population in Java Island, however, psoriasis treatment modalities such as NB-UVB phototherapy, MTX, and biological agents are not equally distributed in this region. Therefore, it is important to identify the HLA-Cw6 allele status and its association with the effectiveness treatment methods available in Indonesia. In this study, $23(25.56 \%)$ psoriasis subjects express HLACw6 allele positivity out of totally 90 subjects. Although 
Table 3. Characteristics of Javanese-Indonesian psoriasis subjects under methotrexate treatment (group B).

\begin{tabular}{|c|c|c|c|c|}
\hline \multirow[t]{2}{*}{ Subject Characteristics } & \multicolumn{2}{|c|}{$\begin{array}{c}\text { HLA-Cw6 } \\
\text { n (\%) }\end{array}$} & \multirow[t]{2}{*}{$p$-value } & \multirow{2}{*}{$\begin{array}{c}\text { Confidence } \\
\text { Interval }\end{array}$} \\
\hline & Positive $(n=11)$ & Negative $(n=34)$ & & \\
\hline \multicolumn{5}{|l|}{ Age at psoriasis onset (years old) } \\
\hline$<40$ & $1(2.22)$ & $13(28.89)$ & $0.132 *$ & $0.031-1.566$ \\
\hline$\geq 40$ & $10(22.22)$ & $21(46.67)$ & & \\
\hline \multicolumn{5}{|l|}{ Gender } \\
\hline Male & $4(8.89)$ & $18(40.00)$ & $0.339^{\dagger}$ & $0.203-1.760$ \\
\hline Female & $7(15.56)$ & $16(35.56)$ & & \\
\hline \multicolumn{5}{|l|}{ Family history } \\
\hline Positive & $2(4.44)$ & $3(6.67)$ & $0.582 *$ & $0.526-6.009$ \\
\hline Negative & $9(20.00)$ & $31(68.89)$ & & \\
\hline \multicolumn{5}{|l|}{ Comorbid diseases } \\
\hline Positive & $3(6.67)$ & $8(17.78)$ & $0.699 *$ & $0.153-2.435$ \\
\hline Negative & $8(17.78)$ & $26(57.78)$ & & \\
\hline \multicolumn{5}{|l|}{ Types of comorbid diseases } \\
\hline Hypertension & $1(9.09)$ & $4(36.36)$ & & \\
\hline Psoriasis arthritis & $2(18.18)$ & $0(0)$ & & \\
\hline Diabetes mellitus & $0(0)$ & $3(27.27)$ & & \\
\hline Congestive heart failure & $0(0)$ & $1(9.09)$ & & \\
\hline \multicolumn{5}{|l|}{ Adverse events } \\
\hline Positive & $2(4.44)$ & $7(15.56)$ & $1.000 *$ & $0.231-3.418$ \\
\hline Negative & $9(20.00)$ & $27(60.00)$ & & \\
\hline \multicolumn{5}{|l|}{ Methotrexate therapeutic response } \\
\hline Good & $6(24.00)$ & $19(76.00)$ & $1.000 *$ & $0.342-2.692$ \\
\hline Poor & $5(25.00)$ & $15(75.00)$ & & \\
\hline
\end{tabular}

${ }^{*}$ Fisher's exact test, ${ }^{\dagger}$ Chi square test.

the study only covered a small size of population, however, it shows that HLA-Cw6 positivity in Javanese people is higher than that in Malay-Indonesian population according to another study.(20) Previous studies with relatively small number of psoriasis populations in Indonesia consistently show that HLA-Cw6 allele expression ranges from 20$25 \% .(10,20)$ Another study reports that positive HLA-Cw6 may vary widely from $14.1 \%-59.1 \%$, and from which only $10.5 \%$ to $77.2 \%$ psoriasis subjects carry the allele.(5) This study result also confirms that HLA-Cw6 allele expression in Asians is lower than that in Europeans or Americans. $(3,5)$

The HLA-Cw6 allele may be correlated with type 1 psoriasis and female gender in Iceland study covering a large number of Caucasian psoriasis populations.(21) In a study with different population, with Indian Tamil psoriasis population, meanwhile, a significant association is found of the HLA-Cw6 allele with female gender and early-onset disease, but not with familial inheritance.(22) The result of our study did not show any statistical significance between the HLA-Cw6 allele expression in Javanese-Indonesian psoriasis subjects and type 1 psoriasis and female gender, and it confirms previous study with the same population. (10) This study result supports the previous studies stating that psoriasis has multiple genetic backgrounds making it able to skip several generations and emerge spontaneously in psoriasis subjects without familial history since familial inheritance is influenced by multiple factors. Besides, HLA-Cw6 is likely not the only genetic factor of psoriasis, including in Javanese-Indonesian psoriasis subjects. $(21,23)$

Comorbid diseases may increase the mortality rate of psoriasis subjects. Cardiovascular diseases and metabolic syndrome are commonly found as the comorbid diseases of psoriasis, and the association of HLA-Cw6 allele with the existence of comorbid diseases is still debatable. It is believed that psoriasis subjects who express HLACw6 allele may have prolonged inflammation because of induction of naive $\mathrm{CD} 8^{+} \mathrm{T}$ cells by this allele to produce IL-17.(6) Furthermore, prolonged inflammation may lead to worsening psoriasis, and the more severe this disease is, the more severe the comorbid diseases are. Therefore, comorbid 
diseases may hinder anti-psoriatic effectiveness. Previous studies show the association of obesity with HLA-Cw6 allele in psoriasis vulgaris cases in Chinese Han population.(24) However, a study in India reports no significant association of metabolic syndrome in child and adult psoriasis with HLA-Cw6 allele expression.(25) Interestingly, our study found that one psoriasis patient with positive HLA-Cw6 status under NB-UVB phototherapy with comorbid disease shows poor therapeutic response. Two subjects in group B who expressed HLA-Cw6 with psoriatic arthritis also show poor MTX therapeutic response. These findings showed the tendency that psoriasis subjects with HLA-Cw6 allele positivity and comorbid diseases may have poor prognosis because of ineffective treatment response. Although there was no significant association of HLA-Cw6 allele status with comorbid diseases in our study, further studies with a large number of populations are necessary to prove this assumption. More investigations are also required to identify the association of comorbid diseases with psoriasis subjects' life style and environmental factors regardless HLA-Cw6 positivity. It is important to taken them into consideration since psoriasis is a multigenic disease with gene-environment interaction. Multigenes may affect the clinical type of psoriasis and comorbid diseases possibilities and HLA-Cw6 is not the main gene associated with this condition in Javanese-Indonesian psoriasis population.

NB-UVB and MTX are the mostly applied psoriasis therapeutic methods in Indonesia, but the association of HLA-Cw6 allele with its treatment responses is still unknown. Ultraviolet B may be proapoptotic and immunosuppressive to $\mathrm{T}$ cells, making HLA-Cw6 unable to present antigen to these cells. This is followed with increasing anti-psoriatic cytokines and decreasing pro-inflammatory cytokines.(26) MTX has a similar effect on NB-UVB in the form of apoptotic effect through DNA synthesis inhibition, however, MTX may cause hematological, gastrointestinal, kidney disorders and teratogenic effect.(13) NB-UVB poses side effects such as erythema, skin burning and photoaging with limited systemic effects (27), thus it may be a safer alternative therapy to MTX. Previous studies reveal that Iceland psoriasis population obtains beneficial effect from sunlight, although there is no statistical difference between the groups that receive NB-UVB and psoralen with UV A.(21) Meanwhile, our finding showed a statistically significant correlations of HLA-Cw6 positivity with NBUVB therapeutic response better than MTX. This good therapeutic effect of NB-UVB was observed in HLA-Cw6 positive subjects without comorbid diseases. Furthermore, psoriasis subjects treated with MTX in this study showed more comorbid diseases and slightly higher PASI score than NB-UVB treated subjects, although all of the subjects are of the same category in the baseline study. This study result shows that HLA-Cw6 allele status without comorbid disease may present a better therapeutic response, especially with NB-UVB treatment method.

Our study has limitation in some matters, including small number of samples of genetic epidemiology, short study duration, and non-further investigated environmental factors that may influence therapeutic responses. Even though this study has several limitations, however this study may present additional scientific information on the clinical use of genetic examination to predict psoriasis therapeutic response in specific Southeast Asian population.

\section{Conclusion}

HLA-Cw6 allele presents good response in female JavaneseIndonesian plaque-type psoriasis subjects treated with NB-UVB therapy without comorbid disease. Meanwhile, HLA-Cw6 allele positivity still shows an association with NB-UVB with better response than with MTX as long as there is no comorbid disease.

\section{Acknowledgements}

We would like to thank our colleagues, Lantip Rujito for his help in compiling this article and Shinta Prima Ardinas for her help in conducting HLA-Cw6 allele examination with patience. This research was funded by Public Service Body's Grant, Universitas Jenderal Soedirman 2019 Number: P/501/UN23/14/PN/2019.

\section{Authors Contribution}

TS and IOP were involved in planning and supervised the work, meanwhile TS, IOP and TO performed the measurements. OPS and FA processed the experimental data, performed the analysis, calculations and statistical analysis. TS drafted the manuscript and designed the figures. TS, IOP, and TO helped in interpreting the results and worked on the manuscript. All authors discussed the results and commented on the manuscript. 


\section{References}

1. Liang Y, Sarkar MK, Tsoi LC, Gudjonsson JE. Psoriasis: a mixed autoimmune and autoinflammatory disease. Curr Opin Immunol. 2017; 49: 1-8. doi: 10.1016/j.coi.2017.07.007.

2. World Health Organization. Global Report on Psoriasis 2016. Geneva: WHO; 2016.

3. Segar D, Karmila IGAAD, Praharsini IGAA, Indira IGAAE. Prevalence and clinical manifestations of patients with psoriasis in RSUP Sanglah from 2017 to 2018. Intisari Sains Medis. 2019; 10: 839-44.

4. Talamonti M, Botti E, Galluzo M, Teoli M, Spallonge G, Bavetta M, et al. Pharmacogenetics of psoriasis: HLA-Cw6 but not LCE3B/3C deletion nor TNFAIP3 polymorphism predisposes to clinical response to interleukin 12/23 blocker ustekinumab. Br J Dermatol. 2013; 169: 458-63.

5. Chen L, Tsai TF. HLA-Cw6 and psoriasis. Br J Dermatol. 2018; 178: 854-62.

6. Gisondi P, Bellinato F, Girolomoni G, Albanesi C. Pathogenesis of chronic plaque psoriasis and its intersection with cardio-metabolic comorbidities. Front Pharmacol. 2020; 11: 117-25.

7. Prinz, JC. Human leukocyte antigen-class I alleles and the autoreactive $\mathrm{T}$ cell response in psoriasis pathogenesis. Front Immunol. 2018; 9: 1-7. doi: 10.3389/fimmu.2018.00954.

8. Chiang CC, Cheng WJ, Korinek M, Lin CY, Hwang TL. Neutrophils in psoriasis. Front Immunol. 2019; 10: 1-12. doi: 10.3389/ fimmu.2019.02376.

9. Badan Pusat Statistik. Kewarganegaraan, Suku Bangsa, Agama, dan Bahasa Sehari-hari Penduduk Indonesia: Hasil Sensus Penduduk Indonesia 2010. Jakarta: Badan Pusat Statistik; 2010.

10. Sylviningrum T, Putranti IO, Sari OP, Arjadi F, Sudibyo ES, Manik SN. Association between HLA-Cw6 allele expression and characteristics of Javanese ethnic psoriasis patients in Indonesia. Med J Indones 2019; 28: 370-4.

11. Burlando M, Russo R, Clapasson A, Carmisciano L, Stecca A, Cozzani E, et al. The HLA-Cw6 dilemma: is it really an outcome predictor in psoriasis patients under biologic therapy? A monocentric retrospective analysis. J Clin Med. 2020; 9: 3140-7.

12. West J, Ogston S, Berg J, Palmer C, Fleming C, Kumar V, et al. HLACw6-positive patients with psoriasis show improved response to methotrexate treatment. Clin Exp Dermatol. 2017; 42: 651-5.

13. Yélamos O, Puig L. Systemic methotrexate for the treatment of psoriasis. Expert Rev Clin Immunol. 2015; 11: 553-63.
14. Wong T, Hsu L, Liao W. Phototherapy in psoriasis: a review of mechanisms of action. J Cutan Med Surg. 2013; 17: 6-12.

15. Ku SH, Kwon WJ, Cho EB, Park EJ, Kim KH, Kim KJ. The association between psoriasis area and severity index and cardiovascular risk factor in korean psoriasis patients. Ann Dermatol. 2016; 28: 360-3.

16. Singh RK, Lee KM, Jose MV, Nakamura M, Umcak D, Farahnik $\mathrm{B}$, et al. The patient's guide to psoriasis treatment. Part 1: UVB Phototherapy. Dermatol Ther (Heidelb). 2016; 6: 307-13.

17. Yan K, Zhang Y, Han L, Huang Q, Zhang Z, Fang X, et al. Safety and efficacy of methotrexate for Chinese adults with psoriasis with and without psoriatic arthritis. JAMA Dermatol. 2019; 155(3): 327 34.

18. Van TN, Van TH, Minh PPT, Trong HN, Van TC, Huu ND, et al. Efficacy of narrow-band UVB phototherapy versus PUVA chemophototherapy for psoriasis in Vietnamese patients. Open Access Maced J Med Sci. 2019; 7: 227-30.

19. Rebała K, Szczerkowska-Dobosz A, Niespodziana K, Wysocka J. Simple and rapid screening for HLA-Cw*06 in Polish patients with psoriasis. Clin Exp Dermatol. 2010; 35: 431-6.

20. Lestari KS, Darwin E, Jacoeb T, Tjong D. The relationship between human leukocyte antigen-cw6 allele and psoriasis vulgaris. Dermatol Reports. 2019; 11(1S): 8054.

21. Gudjónsson JE, Kárason A, Antonsdóttir AA, Rúnarsdóttir EH, Gulcher JR, Stefánsson K, et al. HLA-Cw6-positive and HLACw6-negative patients with psoriasis vulgaris have distinct clinical features. J Invest Dermatol. 2002; 118: 362-5.

22. Indhumathi S, Rajappa M, Chandrashekar L, Ananthanarayanan PH, Thappa DM, Negi VS. The HLA-C*06 allele as a possible genetic predisposing factor to psoriasis in south Indian Tamils. Arch Dermatol Res. 2016; 308: 193-9.

23. Fry L. Psoriasis. Br J Dermatol. 1988; 119: 445-61.

24. Jin Y, Zhang F, Yang S, Kong Y, Xiao F, Hou Y, et al. Combined effects of HLA-Cw6, body mass index and waist-hip ratio on psoriasis vulgaris in Chinese Han population. J Dermatol Sci. 2008; 52: 123-9.

25. Sathishkumar D, George R, Daniel D, Peter JV. Clinical profile of childhood-onset psoriasis and prevalence of HLA-Cw6: a hospital-based study from India. Postgrad Med J. 2015; 91: 309-14.

26. Wolf P, Weger W, Patra V, Gruber-Wackernagel A, Byrne SN. Desired response to phototherapy vs photoaggravation in psoriasis: what makes the difference?. Exp Dermatol. 2016; 25: 937-44.

27. Zhang P, Wu MX. A clinical review of phototherapy for psoriasis. Lasers Med Sci. 2018; 33: 173-80. 